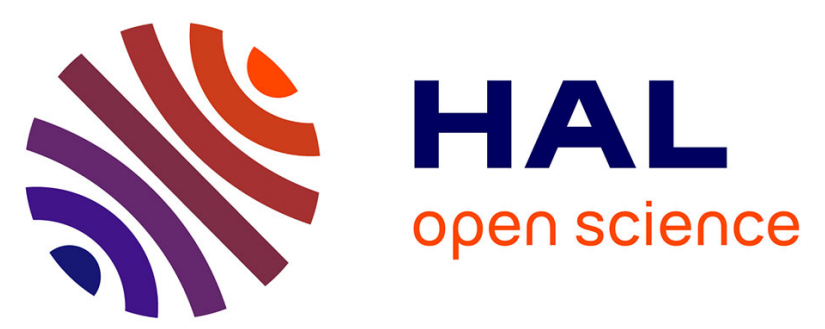

\title{
6 months versus 12 months of adjuvant trastuzumab in early breast cancer (PHARE): final analysis of a multicentre, open-label, phase 3 randomised trial
}

Xavier Pivot, Gilles Romieu, Marc Debled, Jean-Yves Pierga, Pierre Kerbrat, Thomas Bachelot, Alain Lortholary, Marc Espié, Pierre Fumoleau, Daniel Serin, et al.

\section{To cite this version:}

Xavier Pivot, Gilles Romieu, Marc Debled, Jean-Yves Pierga, Pierre Kerbrat, et al.. 6 months versus 12 months of adjuvant trastuzumab in early breast cancer (PHARE): final analysis of a multicentre, open-label, phase 3 randomised trial. The Lancet, 2019, 393 (10191), pp.2591-2598. inserm-02944688

\section{HAL Id: inserm-02944688 https://www.hal.inserm.fr/inserm-02944688}

Submitted on 26 Oct 2021

HAL is a multi-disciplinary open access archive for the deposit and dissemination of scientific research documents, whether they are published or not. The documents may come from teaching and research institutions in France or abroad, or from public or private research centers.
L'archive ouverte pluridisciplinaire HAL, est destinée au dépôt et à la diffusion de documents scientifiques de niveau recherche, publiés ou non, émanant des établissements d'enseignement et de recherche français ou étrangers, des laboratoires publics ou privés.

\section{(ㅇ)(1) $\$$}

Distributed under a Creative Commons Attribution - NonCommerciall 4.0 International 
Title: Final results of the PHARE randomized trial comparing 6 to 12 months of trastuzumab in adjuvant early breast cancer

Xavier Pivot, Gilles Romieu, Marc Debled, Jean-Yves Pierga, Pierre Kerbrat, Thomas Bachelot, Alain Lortholary, Marc Espié, Pierre Fumoleau, Daniel Serin, Jean-Philippe Jacquin, Christelle Jouannaud, Maria Rios, Sophie Abadie-Lacourtoisie, Laurence Venat-Bouvet, Laurent Cany, Stéphanie Catala, David Khayat, Laetitia Gambotti, Iris Pauporté, Celine Faure-Mercier Sophie Paget-Bailly Julie Henriques Jean Marie Grouin, 
Authors degree and title

Prof. Xavier Pivot, M.D, Gilles Romieu, M.D., Marc Debled, M.D., Prof. JeanYves Pierga, M.D., Prof. Pierre Kerbrat, M.D., Thomas Bachelot, M.D., Alain Lortholary, M.D., Marc Espié, M.D., Prof. Pierre Fumoleau, M.D., Daniel Serin, M.D., Jean-Philippe Jacquin, M.D., Christelle Jouannaud, M.D., Maria Rios, M.D., Sophie Abadie-Lacourtoisie, M.D., Prof. Laurence Venat-Bouvet, M.D., Laurent Cany, M.D., Stéphanie Catala, M.D., Prof. David Khayat, M.D., Laetitia Gambotti, M.D., Iris Pauporté, Ph.D., Celine Faure-Mercier Ph.D., Sophie Paget-Bailly Ph.D., Julie Henriques Ph.D., Jean Marie Grouin, Ph.D.

\section{Author Affiliations:}

Centre Paul Strauss, Porte de l'Hopital Strasbourg, France (X.P.); Centre Val d’Aurelle, Montpellier (G.R.); Institut Bergonié, Bordeaux (M.D.); Institut Curie, University Paris Descartes, Paris (J-Y.P.); Centre Eugene Marquis, Rennes (P.K.); Centre Léon Bérard, Lyon (T.B.); Centre Catherine de Sienne, Nantes (A.L.); University Hospital Saint-Louis, Paris (M.E.); Institut Curie, Paris (P.F.); Institut Sainte Catherine, Avignon (D.S.); Institut de Cancérologie Lucien Neuwirth, Saint-Priest-en-Jarez (J-P.J.); Institut Jean Godinot, Reims (C.J.); Centre Alexis Vautrin, Vandoeuvre-les-Nancy (M.R.); Institut de Cancérologie de l'Ouest, Angers (S.A-L.); University Hospital, Limoges (N.T-M.); Clinique Francheville, Périgueux (L.C.); Clinique SaintPierre, Perpignan (S.C.); Clinique Bizet, Paris (D.K.); French National Cancer Institut, Boulogne (L.G.; C.F.M.); Ligue contre le cancer - France, Paris (I.P.); University Hospital, Besancon (S.P.B., J.H.); Department of Statistics, University of Rouen (J.M.G.). 
* Additional investigators of the PHARE trial are listed in the supplementary appendix.

Correspondence to: Xavier Pivot Centre Paul Strauss

3 rue de la porte de l'Hopital

BP 30042

67065 Strasbourg Cedex France

Tel bureau +33388252401

xpivot@strasbourg.unicancer.fr 
Abstract

Background: In 2013, the first premature analysis of the PHARE ('Protocol for Herceptin ${ }^{\circledR}$ as Adjuvant therapy with Reduced Exposure') trial, failed to prove that 6 months of adjuvant trastuzumab was noninferior to 12 months (NCT00381901). This manuscript reports the planned final analysis based on the prespecified number of occurring events. Methods: A total of 3,380 patients with HER2-positive early breast cancer were randomly assigned in this multicentric, unblinded, academic trial, to 12 or 6 months of adjuvant trastuzumab. The randomization was stratified by concomitant or sequential treatment with chemotherapy, estrogen receptor (ER) status and center. The primary objective was noninferiority in the intent-to-treat population in the 6 month arm in terms of disease-free survival (DFS) with a prespecified hazard margin of 1.15. Overall survival (OS) and metastasis-free survival (MFS) were secondary endpoints. Findings: At a median follow-up of 7.5 years, 704 events relevant to DFS were observed. The adjusted hazard ratio (HR) for DFS in the 12- and 6-month arms was $1.08(95 \% \mathrm{Cl} 0.93-1.25 ; \mathrm{p}=0.39)$. The noninferiority margin was included in the $95 \% \mathrm{Cl}$. No differences in effects pertaining to trastuzumab duration were found in any subgroups. For OS and MFS, the adjusted HRs were $1.13(95 \% \mathrm{Cl} 0.92-1.39)$ and $1.15(95 \% \mathrm{Cl}$ 0.96-1.37), respectively. After the completion of trastuzumab, rare adverse events occurred over time and the safety analysis remained similar to the previously published report. Interpretation: The PHARE study failed to prove the noninferiority of 6 months versus 12 months of adjuvant trastuzumab. 
Funding: The PHARE trial was funded by the French National Cancer Institute (www.e-cancer.fr).

Keywords: Trastuzumab, adjuvant treatment, breast cancer, noninferiority trial, randomized trial 
Several large randomized clinical trials have demonstrated that one year of trastuzumab reduces the risk of relapse and death for patients with HER2positive early breast cancer compared to observation (1) (2) (3) (4) (5) (6) (7). This one-year treatment duration has been challenged by several published and ongoing trials. The assessment of a longer 2-year duration of trastuzumab in the HERA trial did not show any significant benefit (8). However, a few studies have assessed a shorter duration of adjuvant trastuzumab, and the one year standard of care has not changed (9) (10)(11) (12)(13). In 2012, the first analysis of the PHARE ('Protocol for Herceptin® as Adjuvant therapy with Reduced Exposure') trial failed to show that 6 months of adjuvant trastuzumab was noninferior to 12 months (9). This first efficacy analysis was requested by the Independent Data Monitoring Committee (IDMC) at a meeting held in May 2011 due to worries regarding negative efficacy signals. Of note, this 2012 analysis was based on a limited number of 394 events and hence was not powered with regard to the initial objective of the trial. The definitive conclusion of the PHARE trial has therefore been pending a final analysis based on the prespecified number of disease-free survival (DFS) events, which is the aim of the current manuscript.

\section{Patients and methods}

The study design, eligibility criteria, patient characteristics, and treatment compliance have been described previously and are summarized herein (9). 


\section{Study design}

PHARE is an open-label phase 3 randomized noninferiority trial of patients with HER2-positive early breast cancer comparing 6 versus 12 months of trastuzumab treatment concomitant with or subsequent to standard neoadjuvant or adjuvant chemotherapy. The trial was sponsored by the French National Cancer Institute (INCa), approved by the Central Ethics Committee on May 15, 2006, and registered at http://www.clinicaltrials.gov (NCT00381901). It was conducted in compliance with the principles of Good Clinical Practice (GCP) and the Declaration of Helsinki. An IDMC assessed and monitored the trial. After the completion of trastuzumab treatment, patients were followed up every 3 months during the first two years, every 6 months until the fifth year and then every year thereafter. Two consecutive missing reports qualified the patients in the group lost to follow-up and they were censored at the date of their last news.

Randomization: A central randomization procedure was set up using TenAlea web-based software (http://fr.tenalea.net). Eligible patients were randomized to the two durations of trastuzumab between the third and sixth months of adjuvant trastuzumab. Using a minimization algorithm, 1:1 randomization was stratified on concomitant versus sequential administration of trastuzumab with chemotherapy and positive versus negative tumor ER status.

Endpoints: The primary endpoint was DFS, defined as the time from randomization to the first occurrence of any of the following events: local, regional or distant relapse; contralateral breast cancer; second nonbreast malignant disease; or death from any cause. Patients alive without any 
predefined event were censored at the time of the last assessment. Secondary efficacy endpoints included overall survival (OS) (the time from randomization to death from any cause) and metastasis-free survival (MFS) (the time from randomization to distant relapse or death from any cause, whichever occurred first).

Safety endpoints and compliance were previously reported, as was a dedicated subanalysis of cardiac safety $(9,14)$. This analysis assessed only cardiotoxicity events occurring after trastuzumab completion defined as a decrease in the left ventricular ejection fraction (LVEF) below 50\% regardless of baseline LVEF and an absolute decrease of $10 \%$ from baseline with an LVEF below $50 \%$.

\section{Statistical analyses:}

The hypothesis of the PHARE trial is that 6 months of adjuvant trastuzumab is not inferior to 12 months in terms of DFS.

The prespecified noninferiority margin in the protocol was set to $15 \%$ in relative terms, corresponding to a hazard ratio $(\mathrm{HR})$ of 1.15 . In absolute terms, this difference corresponds to a $2 \%$ difference in DFS at 2 years, i.e., from $85 \%$ (as estimated in the HERA trial) to $83 \%$, assuming an exponential survival distribution (1).

In 2011, due to a negative efficacy signal associated with the 6-month group, the IDMC recommended stopping patient accrual and continuing patient follow-up until July 2012, which corresponds to 4 years of enrollment and a minimum of 2 years of follow-up before releasing the data. Hence, an 
amended statistical plan was written in August 2011 prespecifying this first analysis requested by the IDMC at an unplanned interim stage and planning the final analysis based on an adequate number of events. With the goal of preserving the noninferiority margin of $1.15,680$ DFS events were required in the final analysis to claim noninferiority with $80 \%$ power at the $5 \%$ two-sided significance level.

This final analysis of the PHARE trial was based on the database lock on December 14, 2017. This Database is preserved at the French National Cancer Institute.

The main analyses were performed in the intent-to-treat (ITT) set. A sensitivity analysis was performed in the per protocol (PP) set defined as the ITT subset of patients who actually received trastuzumab for 6 months $+/-1$ month in the 6-month randomized group and for 12 months +/- 2 months in the 12-month randomized group.

All survival rates for each time-to-event endpoint were estimated using the Kaplan-Meier method and the 95\% confidence interval $(\mathrm{Cl})$. Hazard ratios (HRs) for the treatment effect (6 versus 12 months), along with their $95 \%$ confidence intervals $(\mathrm{Cls})$, were estimated using the proportional hazards Cox model adjusted for the stratification factors, including ER status (negative versus positive) and chemotherapy-trastuzumab timing (sequential versus concomitant). The same analyses were performed in a multivariate Cox model adjusted for treatment arm (6 versus 12 months), ER status (negative versus positive), progesterone receptor (PgR) status (negative 
versus positive), nodal status (negative versus positive), tumor size $(<2 \mathrm{~cm}$ versus $\geq 2 \mathrm{~cm}$ ) and chemotherapy-trastuzumab timing (sequential versus concomitant).

Proportional hazards were tested using Schoenfeld residuals (15), and smoothed HRs are presented. When the proportional hazards assumption did not hold, the restricted mean survival time (RMST) was estimated within each arm, and the difference and ratio of RMST were estimated, along with the $95 \% \mathrm{Cl}$, by bootstrap simulation (16) (17). A positive difference or a ratio above 1 indicates a result favoring the 12-month arm.

The consistency of the treatment effect was assessed across prognostic factor subgroups, and the treatment by subgroup interaction was tested in a Cox model adjusted for each prognostic factor separately. The potential influence of each prognostic factor on the first DFS was also assessed in a Cox model adjusted for each prognostic factor separately.

\section{Role of the sponsor and the steering committee}

Data collection was under the responsability of the sponsor. The analysis and interpretation of the results in this paper were performed independently under the auspices of the PHARE executive committee. JMG and XP led the writing and analysis of the paper. The decision to submit was entrusted to XP.

\section{Results}

Patients were randomized from May 30, 2006, to July 09, 2010 (figure 1). A total of 234 patients were lost to follow-up over the time and their distribution 
was well balanced between the two arms. At the time of the present analysis, the median follow-up for the 3,380 patients was 7.5 years from randomization (IQR 5.3 - 8.8). Patient, disease and treatment characteristics were well balanced between the 2 treatment arms, as previously reported (table 1).

\section{Primary objective}

A total of 704 DFS events, including 345 (20.4\%) and 359 (21.2\%) in the 12and 6-month arms, respectively, were reported. In the 12- and 6-month arms, there were 163 and 187 distant recurrences, 53 and 60 local-regional relapses, 27 and 33 contralateral breast cancers, 24 and 18 deaths, and 78 and 61 second primary malignancies, respectively. The HR adjusted for stratification factors was $1.08(95 \% \mathrm{Cl} 0.93-1.25$; figure $2 \mathrm{~A})$. Since the prespecified noninferiority margin of 1.15 was included in the $\mathrm{Cl}$, the results were inconclusive regarding the noninferiority hypothesis $(p=0.39)$. The survival estimates at 3, 5 and 7 years were: $92.2 \%$ versus $89.3 \%, 86.2 \%$ versus $84.2 \%$ and $82.3 \%$ versus $80.6 \%$ in the in 12 - and 6 -months arms, respectively. Subgroup analyses showed a consistent treatment effect (figure 3).

The proportional hazard assumption of the Cox model was tested based on Schoenfeld residuals, and this assumption was rejected $(p=0.004)$. The graph of smoothed HRs over time supported this nonproportionality (figure 2D). Of interest, a Cox model including time as a time-dependent covariate enabled the estimation of the HR over the first 2 years. The HR of DFS events over the first two years was $1.43(95 \% \mathrm{Cl} 1.12-1.84)$, indicating that more DFS events occurred early in the 6-month arm than in the 12-month 
arm. Another summary of the survival curve differences was provided by the difference in the RMST (12 months minus 6 months) and the RMST ratio (12 months over 6 months) up to 9.5 years; these values were 0.17 years $(95 \%$ $\mathrm{Cl} 0.2-0.37)$ and $1.02(95 \% \mathrm{Cl} 1.00-1.04)$, respectively, and favored the $12-$ month group.

In the PP analysis, $273 / 1,372$ (19.9\%) and 282/1,356 (20.8\%) DFS events occurred in the 12- and 6-month groups, respectively. The estimated HR adjusted for the stratification factors was $1.10(95 \% \mathrm{Cl}$ 0.93-1.30; supplementary figure 1).

\section{Secondary objectives}

\section{Overall survival}

A total of $170(10.1 \%)$ and $186(11.0 \%)$ deaths occurred in the 12- and 6month groups, respectively (figure 2B). The estimated HR was $1.13(95 \% \mathrm{Cl}$ 0.92-1.39). Subgroup analysis revealed a consistent treatment effect. A graph of smoothed HRs over time is shown (figure $2 \mathrm{E}$ ), and the proportional hazards assumption was once again rejected $(p=0.006)$.

\section{Metastasis-free survival (MFS)}

The distribution of MFS event types showed that 224 (13.3\%) and 249 (14.7\%) patients in the 12- and 6-month arms, respectively, experienced distant recurrence as the first event; the estimated $\mathrm{HR}$ was $1.15(95 \% \mathrm{Cl}$ 
0.96-1.37; figure 2C). Subgroup analysis showed a consistent treatment effect. A graph of smoothed HRs over time is presented (figure 2F), and the proportional hazards assumption was again rejected $(p=0.013)$.

\section{Prognostic factor analyses}

An exploratory univariate Cox proportional hazards model was used to analyze the primary endpoint DFS and the secondary endpoints OS and MFS. Nodal status, ER status, PgR status and tumor size were significantly related to outcome. Additionally, the interaction test in the multivariate model revealed no significant heterogeneity related to the survival endpoints. The estimated HRs adjusted for all previous significant factors and chemotherapy-trastuzumab timing for the comparison of the 6- and 12-month arms in terms of DFS, OS and MFS were $1.07(95 \% \mathrm{Cl} 0.92-1.24), 1.07$ (95\% Cl 0.86-1.32) and 1.13 (95\% Cl 0.94-1.36), respectively.

\section{Safety}

After the completion of trastuzumab, rare safety events occurred over time. Since the previous publication, no additional cases of heart failure have occurred, and only 3 cases in which LVEF decreased $<50 \%$ have been reported in the 12-month arm(14). No change regarding the cardiac safety comparison appeared with this longer follow-up analysis.

\section{Discussion}


The first analysis of the PHARE trial in 2012 failed to demonstrate noninferiority between the 6-month and 12-month treatment durations (9). This first analysis, which was performed based on the suggestion of the IDMC, included a limited number of DFS events, and the heterogeneity of the treatment effect regarding ER status and trastuzumab-chemotherapy timing complicated the interpretation of the findings (18). This first analysis was inconclusive and did not rule out the possibility that 6 months of adjuvant trastuzumab might be noninferior to 12 months of treatment. Because the PHARE trial was interrupted by the IDMC at an advanced stage of accrual, the revised statistical plan preserved the opportunity to produce a valid conclusion. The preservation of the initial statistical hypothesis, with an adaptation of the expected number of events due to a refined estimate of DFS rates (provided by a longer follow-up of trials with one year of adjuvant trastuzumab), allowed for a definitive conclusion.

In this final analysis, the $95 \% \mathrm{Cl}$ included the noninferiority margin, and the PHARE trial failed to demonstrate noninferiority between 6 and 12 months of trastuzumab. In this final analysis, the heterogeneity of the therapeutic effects completely disappeared, confirming the wise policy of not guiding treatment based on subgroup analyses. Especially, the treatment effect was homogeneous addressing the trastuzumab chemotherapy timing (sequential versus concurrent administration modalities). With longer follow-up, a limited number of safety events were reported, and the previously reported comparison of the safety 
profiles in the 6- and 12-month arms did not change (9) (14). The risk/benefit analysis favored 12 months of adjuvant trastuzumab for all patients with HER2-positive early breast cancer.

The lack of stringent criteria for defining the acceptable noninferiority margin complicates the design of such trials and represents obvious limitations. The noninferiority margin of 1.15 was chosen in the PHARE trial as an increase of $15 \%$ on the HR scale could still be considered acceptable. On the other hand, the PERSEPHONE study (NCT00712140), using a similar design with the same clinical endpoint, included a prespecified margin of 1.29 on the HR scale to define noninferiority (13). The results comparing the 12- versus 6-month trastuzumab arms are similar at 1.08 (95\% Cl 0.93-1.25) and 1.07 (90\% CI 0.93-1.24) for the PHARE and PERSEPHONE studies, respectively. The discordant conclusions are explained by a slightly different statistical prespecified endpoint boundary. Of note, if a noninferiority margin of 1.29 instead of 1.15 had been chosen, noninferiority could have been claimed in the PHARE trial. These considerations refer more generally to the debate on the determination of the noninferiority margin. The FDA guidance on noninferiority trials, which was issued after the design of the PHARE trial, is perhaps one of the most illuminating texts on this topic (19). This guideline suggests defining a noninferiority margin to preserve a fraction of the reference treatment effect estimated in historical trials. This approach represents a true improvement in defining noninferiority and equivalence margins. Nevertheless, the acceptable magnitude of preservation is a subject of 
debate. The choice of the noninferiority margin will remain inherently controversial, especially in the context of oncology trials, where the primary outcome is survival and any additional deaths could be considered unacceptable, thereby throwing into question the very feasibility of noninferiority trials.

Another issue brought up by the PHARE findings that might have occurred in other trials but perhaps not been sufficiently discussed or noted is the lack of validity of the proportional hazards assumption. The utilization of the overall HR to compare survival curves and assess the noninferiority hypothesis might be controversial. In the PHARE trial, the smoothed HRs obviously favored the 12-month arm over the first two years, but this benefit seemed to vanish beyond the third year. Noninferiority of the 6-month versus the 12-month regimen cannot be claimed during the first two years, with an estimated HR over this period clearly favoring the 12-month arm $(1.43 ; 95 \% \mathrm{Cl} 1.12-1.84)$. This observation underlines the early benefit of trastuzumab and could single-handedly legitimately dismiss the need for further investigations. The lack of proportional hazards precludes the use of an overall HR, and another statistical summary is needed. One option would be to consider the survival probability at a specific late time-point. However, this approach does not capture the profile of events over time. An alternative is to use the RMST to summarize the mean survival time of all patients followed up to 9.5 years. The 0.17-year absolute difference in RMST (0.98 relative difference) represents a $2 \%$ relative difference favoring the 12-month arm over the 6-month arm. 
All these statistical considerations consistently favored the 12-month arm, although the magnitude of the difference between arms was limited. Without any safety concerns related to adjuvant trastuzumab, a pharmaco-economic model of the cost savings with a shorter treatment duration seems to be the criterion supporting the decrease in duration. The emergence of biosimilars for trastuzumab will reduce the overall cost and may decrease interest in reducing the trastuzumab treatment duration (20) (21) (22).

\section{Conclusion}

In the PHARE trial, all the statistical investigations favored the 12-month arm. The PHARE trial definitively failed to demonstrate the noninferiority of 6 months of adjuvant trastuzumab, and 12 months might remain the standard of care. Taking into account that the recent promising results obtained with trastuzumab-Emtansine (T-DM1) in the treatment of patients with residual invasive disease after completion of neoadjuvant therapy with a tratuzumab containing regimen, may dramatically change the current treatment strategy in this population (23). Nevertheless, the conclusions reported by PHARE and PERSEPHONE trials questioned our ability to address a strategy aimed to reduce therapy. The discordant conclusions reached by both studies based on similar results reflect the difficulty in reaching a consensus on acceptable or reasonable differences in efficacy to support a reduction in exposure. 
Acknowledgments:

We thank Andrew Kramar for his input in the statistical analysis in 2013.

We thank all patients who gave their consent to be included in the study and the staff of the French National Cancer Institute (INCa) for their assistance with all aspects of conducting the trial. We thank the American Journal Expert (601 W Main St, Durham, North Carolina, 27701, USA) for their support in revising the English.

Author contributions

Study design: XP IP GM PK PF DK

Collection of data IP CFM AK JH SP XP JMG

Statistical analysis XP AK JH SP JMG

Interpretation of results: XP GR MD JYP PK TB AL ME PF DS JPJ CJ MR SAL NT LC SC DK IP CFM SP JH AK JMG

Writing XP JMG

Reading and review GR MD JYP PK TB AL ME PF DS JPJ CJ MR SAL NT LC SC DK IP CFM SP JH AK

Manuscript Approval XP GR MD JYP PK TB AL ME PF DS JPJ CJ MR SAL NT LC SC DK IP CFM SP JH AK JMG

Conflicts of interest

GR, MD, JYP, PK, TB, AL, DS, JPJ, CJ, MR, SAL, LVB, LC, SC, SP, JH, and JG declare no conflicts of interest; XP has been a consultant for AMGEN and SAMSUNG BioEpis; XP, ME and PF have been consultants for Roche and have received honorariums; CFM and LG are employed by the sponsor; and IP and DK were previously employed by the sponsor. 


\section{Bibliography}

1. Piccart-Gebhart MJ, Procter M, Leyland-Jones B, Goldhirsch A, Untch M, Smith I, et al. Trastuzumab after adjuvant chemotherapy in HER2-positive breast cancer. N Engl J Med. 2005;353(16):1659-72.

2. Romond EH, Perez EA, Bryant J, Suman VJ, Geyer CE, Jr., Davidson NE, et al. Trastuzumab plus adjuvant chemotherapy for operable HER2-positive breast cancer. N Engl J Med. 2005;353(16):1673-84.

3. Slamon D, Eiermann W, Robert N, Pienkowski T, Martin M, Press M, et al. Adjuvant trastuzumab in HER2-positive breast cancer. N Engl J Med. 2011;365(14):1273-83.

4. Smith I, Procter M, Gelber RD, Guillaume S, Feyereislova A, Dowsett M, et al. 2-year follow-up of trastuzumab after adjuvant chemotherapy in HER2positive breast cancer: a randomised controlled trial. Lancet. 2007;369(9555):29-36.

5. Perez EA, Romond EH, Suman VJ, Jeong JH, Davidson NE, Geyer CE, Jr., et al. Four-year follow-up of trastuzumab plus adjuvant chemotherapy for operable human epidermal growth factor receptor 2-positive breast cancer: joint analysis of data from NCCTG N9831 and NSABP B-31. J Clin Oncol. 2011;29(25):3366-73.

6. Perez EA, Romond EH, Suman VJ, Jeong JH, Sledge G, Geyer CE, Jr., et al. Trastuzumab plus adjuvant chemotherapy for human epidermal growth factor receptor 2-positive breast cancer: planned joint analysis of overall survival from NSABP B-31 and NCCTG N9831. J Clin Oncol. 2014;32(33):3744-52.

7. Cameron D, Piccart-Gebhart MJ, Gelber RD, Procter M, Goldhirsch A, de Azambuja E, et al. 11 years' follow-up of trastuzumab after adjuvant chemotherapy in HER2-positive early breast cancer: final analysis of the HERceptin Adjuvant (HERA) trial. Lancet. 2017;389(10075):1195-205.

8. Goldhirsch A, Gelber RD, Piccart-Gebhart MJ, de Azambuja E, Procter M, Suter TM, et al. 2 years versus 1 year of adjuvant trastuzumab for HER2-positive breast cancer (HERA): an open-label, randomised controlled trial. Lancet. 2013;382(9897):1021-8.

9. Pivot X, Romieu G, Debled M, Pierga JY, Kerbrat P, Bachelot T, et al. 6 months versus 12 months of adjuvant trastuzumab for patients with HER2positive early breast cancer (PHARE): a randomised phase 3 trial. Lancet Oncol. 2013;14(8):741-8.

10. Conte P, Frassoldati A, Bisagni G, Brandes AA, Donadio M, Garrone O, et al. Nine weeks versus 1 year adjuvant trastuzumab in combination with chemotherapy: final results of the phase III randomized Short-HER studydouble dagger. Ann Oncol. 2018;29(12):2328-33.

11. Mavroudis D, Saloustros E, Malamos N, Kakolyris S, Boukovinas I, Papakotoulas $\mathrm{P}$, et al. Six versus 12 months of adjuvant trastuzumab in combination with dose-dense chemotherapy for women with HER2-positive 
breast cancer: a multicenter randomized study by the Hellenic Oncology Research Group (HORG). Ann Oncol. 2015;26(7):1333-40.

12. Joensuu H, Fraser J, Wildiers H, Huovinen R, Auvinen P, Utriainen M, et al. Effect of Adjuvant Trastuzumab for a Duration of 9 Weeks vs 1 Year With Concomitant Chemotherapy for Early Human Epidermal Growth Factor Receptor 2-Positive Breast Cancer: The SOLD Randomized Clinical Trial. JAMA Oncol. 2018;4(9):1199-206.

13. Earl H., Hiller L., Vallier A.L., Loi S., Howe D., Higgins H., et al. PERSEPHONE: 6 versus 12 months of adjuvant trastuzumab in patients with positive early breast cancer: randomized phase 3 non-inferiority trial with definitive 4-year desease free survival results. American Society of Clinical Oncology (ASCO) Annual Meeting. 2018.

14. Pivot X, Suter T, Nabholtz JM, Pierga JY, Espie M, Lortholary A, et al. Cardiac toxicity events in the PHARE trial, an adjuvant trastuzumab randomised phase III study. Eur J Cancer. 2015;51(13):1660-6.

15. Grambsch P, Therneau T. Proportional hazards test and diagnostics based on weighted residuals. Biometrika. 1994;81:515-26.

16. Royston P, Parmar MK. The use of restricted mean survival time to estimate the treatment effect in randomized clinical trials when the proportional hazards assumption is in doubt. Stat Med. 2011;30(19):2409-21. 17. Andersen PK, Hansen MG, Klein JP. Regression analysis of restricted mean survival time based on pseudo-observations. Lifetime Data Anal. 2004;10(4):335-50.

18. Pivot X. Adjuvant chemotherapy for local relapse breast cancer. Lancet Oncol. 2014;15(2):125-6.

19. Administration FaD, Services UDoHaH. Center for Biologics Evaluation and Research (CBER), Center for Drug Evaluation and Research (CDER). Guidance for industry Non-inferiority clinical trials. http://wwwfdagov/downloads/Drugs//Guidances/UCM201240pdf. 2014. 20. Stebbing J, Baranau Y, Baryash V, Manikhas A, Moiseyenko V, Dzagnidze G, et al. CT-P6 compared with reference trastuzumab for HER2-positive breast cancer: a randomised, double-blind, active-controlled, phase 3 equivalence trial. Lancet Oncol. 2017;18(7):917-28.

21. Pivot X, Bondarenko I, Nowecki Z, Dvorkin M, Trishkina E, Ahn JH, et al. Phase III, Randomized, Double-Blind Study Comparing the Efficacy, Safety, and Immunogenicity of SB3 (Trastuzumab Biosimilar) and Reference Trastuzumab in Patients Treated With Neoadjuvant Therapy for Human Epidermal Growth Factor Receptor 2-Positive Early Breast Cancer. J Clin Oncol. 2018;36(10):96874.

22. von Minckwitz G, Colleoni M, Kolberg HC, Morales S, Santi P, Tomasevic Z, et al. Efficacy and safety of ABP 980 compared with reference trastuzumab in women with HER2-positive early breast cancer (LILAC study): a randomised, double-blind, phase 3 trial. Lancet Oncol. 2018;19(7):987-98.

23. von Minckwitz G, Huang CS, Mano MS, Loibl S, Mamounas EP, Untch M, et al. Trastuzumab Emtansine for Residual Invasive HER2-Positive Breast Cancer. N Engl J Med. 2019;380(7):617-28. 
Table 1: Baseline patient, disease, and treatment characteristics

\begin{tabular}{|c|c|c|}
\hline & $\begin{array}{l}\text { 12-month group } \\
\qquad(n=1,690)\end{array}$ & $\begin{array}{l}\text { 6-month group } \\
(n=1,690)\end{array}$ \\
\hline \multicolumn{3}{|l|}{ Age (years) } \\
\hline$<35$ & $62 \quad(3.7 \%)$ & $66 \quad(3.9 \%)$ \\
\hline $35-49$ & $538 \quad(31.8 \%)$ & $528 \quad(31.2 \%)$ \\
\hline $50-59$ & $514 \quad(30.4 \%)$ & $545 \quad(32.2 \%)$ \\
\hline$\geq 60$ & $576 \quad(34.1 \%)$ & $551 \quad(32.6 \%)$ \\
\hline $\begin{array}{l}\text { Age, median } \\
\text { (range) }\end{array}$ & $54 \quad(21-86)$ & $55 \quad(23-85)$ \\
\hline \multicolumn{3}{|l|}{ Nodal status } \\
\hline Negative & $927 \quad(55.4 \%)$ & $915 \quad(54.7 \%)$ \\
\hline 1-3 positive nodes & $502 \quad(30.0 \%)$ & $506 \quad(30.2 \%)$ \\
\hline$>3$ positive nodes & $(14.6 \%)$ & $253(15.1 \%)$ \\
\hline Missing data & 17 & 16 \\
\hline \multicolumn{3}{|l|}{ Tumor size $(\mathrm{cm})$} \\
\hline$<2$ & $742 \quad(44.9 \%)$ & $703 \quad(42.5 \%)$ \\
\hline$\geq 2-<5$ & $734 \quad(44.4 \%)$ & $753(45.6 \%)$ \\
\hline$\geq 5$ & $178 \quad(10.8 \%)$ & $197(11.9 \%)$ \\
\hline Missing data & 36 & 37 \\
\hline \multicolumn{3}{|c|}{ Scarff -Bloom-Richardson grade } \\
\hline I & $52 \quad(3.1 \%)$ & $54 \quad(3.3 \%)$ \\
\hline II & $679 \quad(41.0 \%)$ & $672(40.9 \%)$ \\
\hline III & $925 \quad(55.9 \%)$ & $918 \quad(55.8 \%)$ \\
\hline Missing data & 34 & 46 \\
\hline \multicolumn{3}{|c|}{ Estrogen receptor status } \\
\hline Negative & $715 \quad(42.3 \%)$ & $695 \quad(41.1 \%)$ \\
\hline Positive & $975 \quad(57.7 \%)$ & $995 \quad(58.9 \%)$ \\
\hline \multicolumn{3}{|c|}{ Progesterone receptor status } \\
\hline Negative & $969 \quad(57.6 \%)$ & $986 \quad(58.4 \%)$ \\
\hline
\end{tabular}




\begin{tabular}{|c|c|c|c|c|c|}
\hline Positive & 712 & $(42.4 \%)$ & & 701 & $(41.6 \%)$ \\
\hline Missing data & 9 & & & 3 & \\
\hline Hormone (estrogen and & esteron & e) recepto & tus & & \\
\hline Negative & 670 & $(39.6 \%)$ & & 650 & $(38.5 \%)$ \\
\hline Positive & 1,020 & $(60.4 \%)$ & & 1,040 & $(61.5 \%)$ \\
\hline Tumor location & & & & & \\
\hline Right & 818 & $(48.4 \%)$ & & 800 & $(47.3 \%)$ \\
\hline Left & 860 & $(50.9 \%)$ & & 872 & $(51.6 \%)$ \\
\hline Both & 12 & $(0.7 \%)$ & & 18 & $(1.1 \%)$ \\
\hline HER2 test results & & & & & \\
\hline IHC HER2+++ & 1,539 & $(91.1 \%)$ & & 1,546 & $(91.5 \%)$ \\
\hline $\begin{array}{l}\text { IHC HER2++, } \\
\text { FISH+ }\end{array}$ & 111 & $(6.6 \%)$ & & 106 & $(6.3 \%)$ \\
\hline $\begin{array}{l}\text { IHC HER2++, } \\
\text { CISH+ }\end{array}$ & 38 & $(2.2 \%)$ & & 37 & $(2.2 \%)$ \\
\hline FISH+ & 2 & $(0.1 \%)$ & & 1 & $(0.1 \%)$ \\
\hline Types of chemotherapy & & & & & \\
\hline Taxane and anthracycline & 1,249 & $(73.9 \%)$ & & 1,229 & $(72.7 \%)$ \\
\hline Anthracycline only & 268 & $(15.9 \%)$ & & 262 & $(15.5 \%)$ \\
\hline Taxane only & 171 & $(10.1 \%)$ & & 196 & $(11.6 \%)$ \\
\hline $\begin{array}{l}\text { Without taxane or } \\
\text { anthracycline }\end{array}$ & 2 & $(0.1 \%)$ & & 3 & $(0.2 \%)$ \\
\hline Timing of chemotherapy & trastuz & umab adm & tratio & & \\
\hline Sequential & 718 & $(42.5 \%)$ & 729 & & $(43.1 \%)$ \\
\hline Concomitant & 972 & $(57.5 \%)$ & 961 & & $(56.9 \%)$ \\
\hline $\begin{array}{l}\text { Data are presented as n (\% } \\
\text { FISH=fluorescent in situ hy }\end{array}$ & $\begin{array}{l}\text { ess othe } \\
\text { zation. }\end{array}$ & $\begin{array}{l}\text { erwise stat } \\
\mathrm{CISH}=\text { chro }\end{array}$ & $\begin{array}{l}\mathrm{IC}=\mathrm{im} \\
\text { nic in }\end{array}$ & $\begin{array}{l}\text { ohistocl } \\
\text { hybridi }\end{array}$ & $\begin{array}{l}\text { hemistry. } \\
\text { ization. }\end{array}$ \\
\hline
\end{tabular}


Figure 1: Flow diagram of the PHARE Trial

\begin{tabular}{|c|}
\hline $\begin{array}{c}\text { Primary Treatment } \\
\text { Local assessment of HER2 status } \\
\text { Surgery and (neo) adjuvant chemotherapy with trastuzumab } \\
+/- \text { radiation and hormone therapy }\end{array}$ \\
\hline $\begin{array}{c}\text { Randomization } \\
\text { Between the 3rd and 6th months of trastuzumab } \\
\text { Between May 30, 2006, and July 31, } 2010\end{array}$ \\
\hline 3,384 patients \\
\hline
\end{tabular}

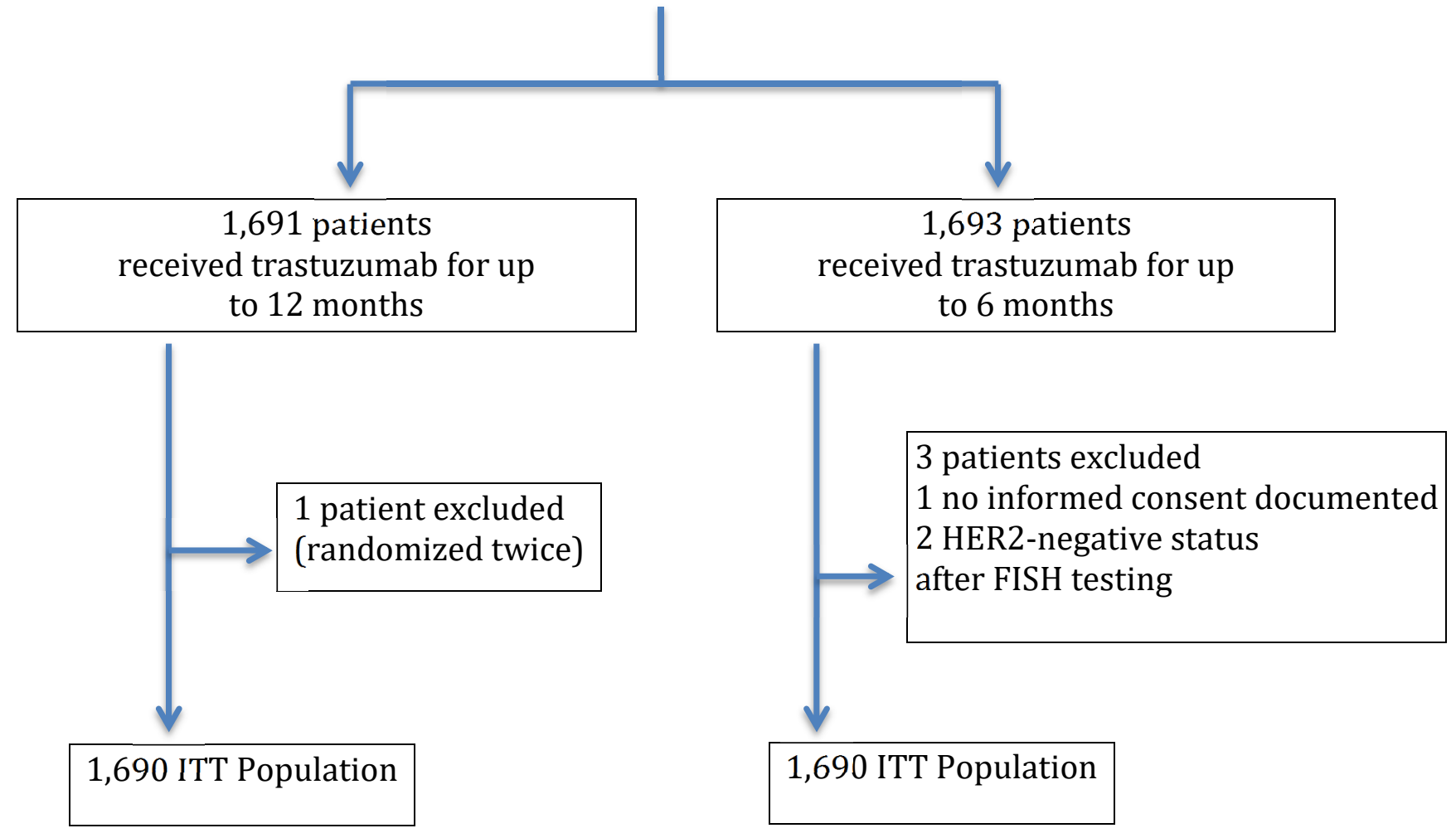


Figure 2A: Disease-free survival according to trastuzumab duration

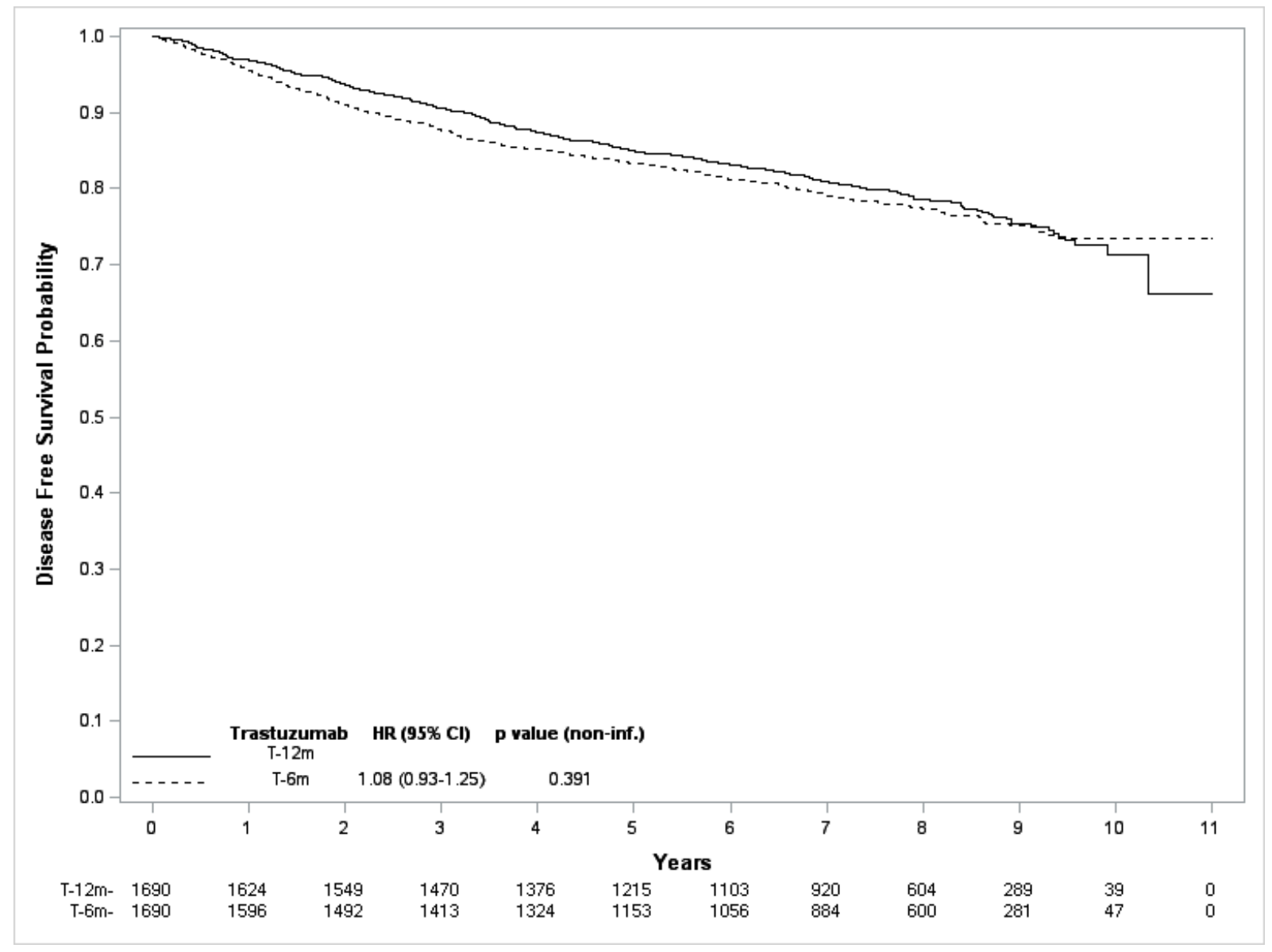

adjusted for stratification factors: estrogen receptor status (+ versus -) and chemotherapy (concomitant versus sequential) 
Figure 2B: Overall survival according to trastuzumab duration

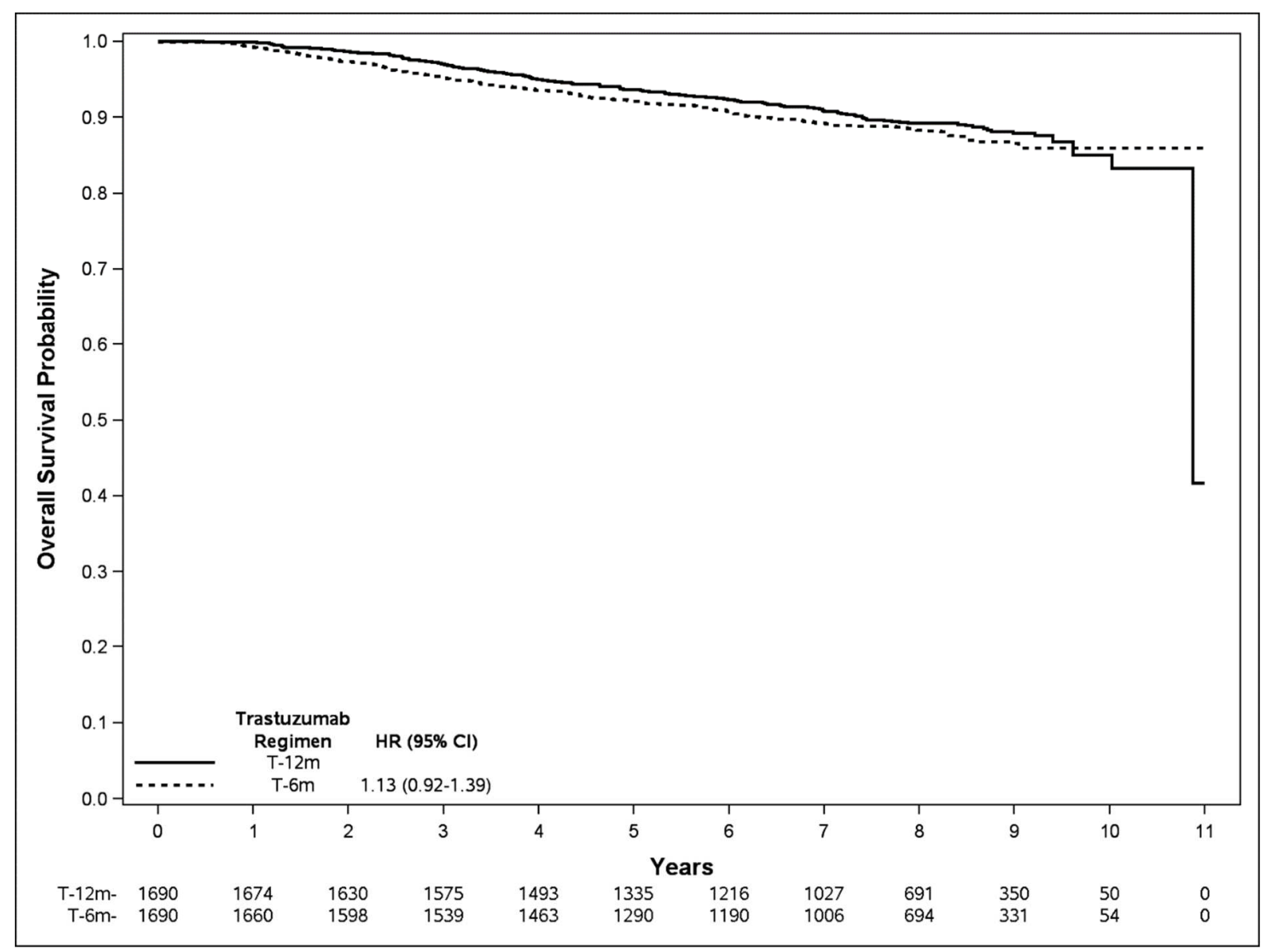

adjusted for stratification factors: estrogen receptor status (+ versus -) and chemotherapy (concomitant versus sequential) 
Figure 2C: Metastasis-free survival according to trastuzumab duration

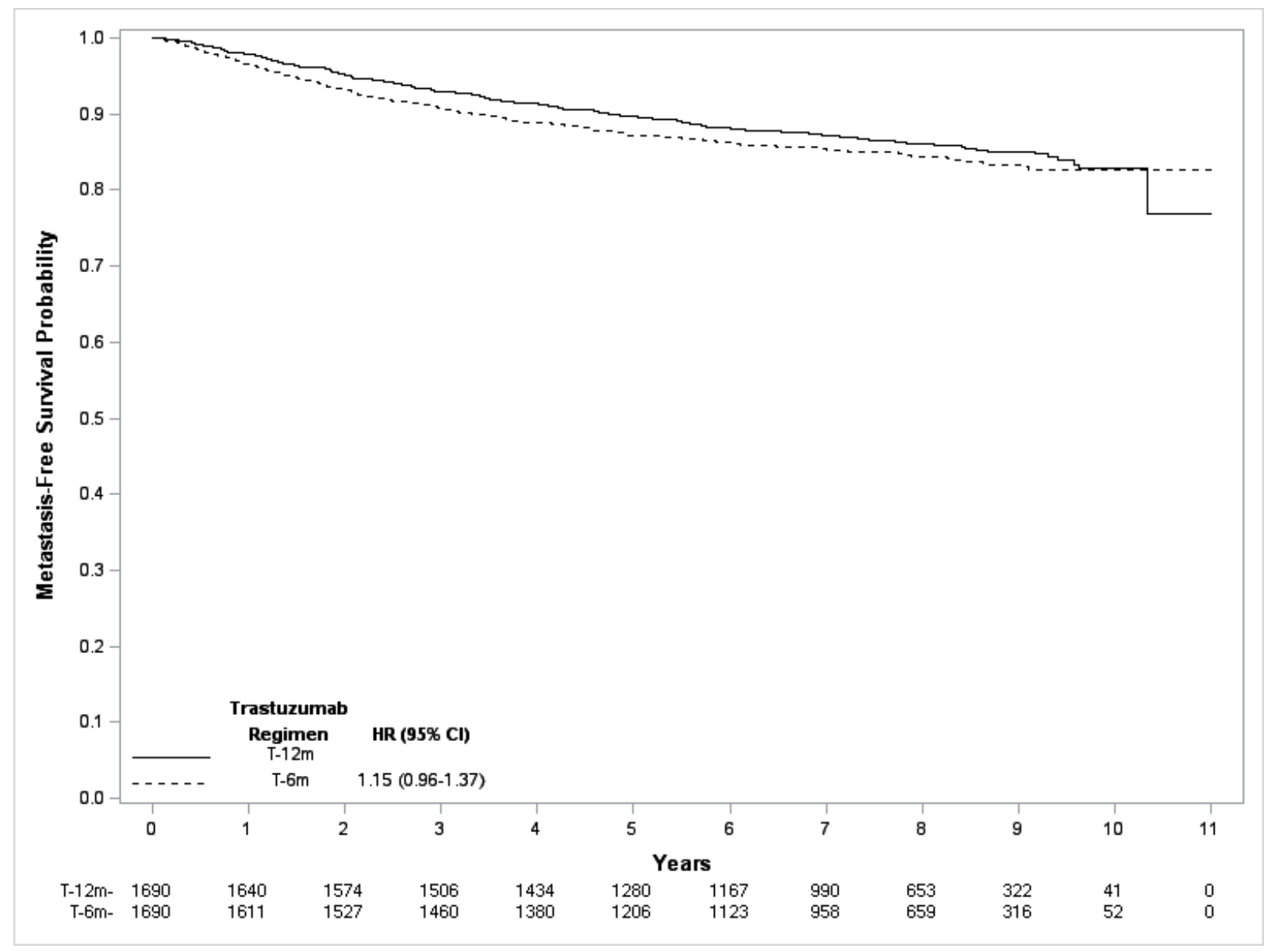

adjusted for stratification factors: estrogen receptor status (+ versus -) and chemotherapy (concomitant versus sequential) 
Figure 2D: Smoothed hazard ratios over time for disease-free survival events

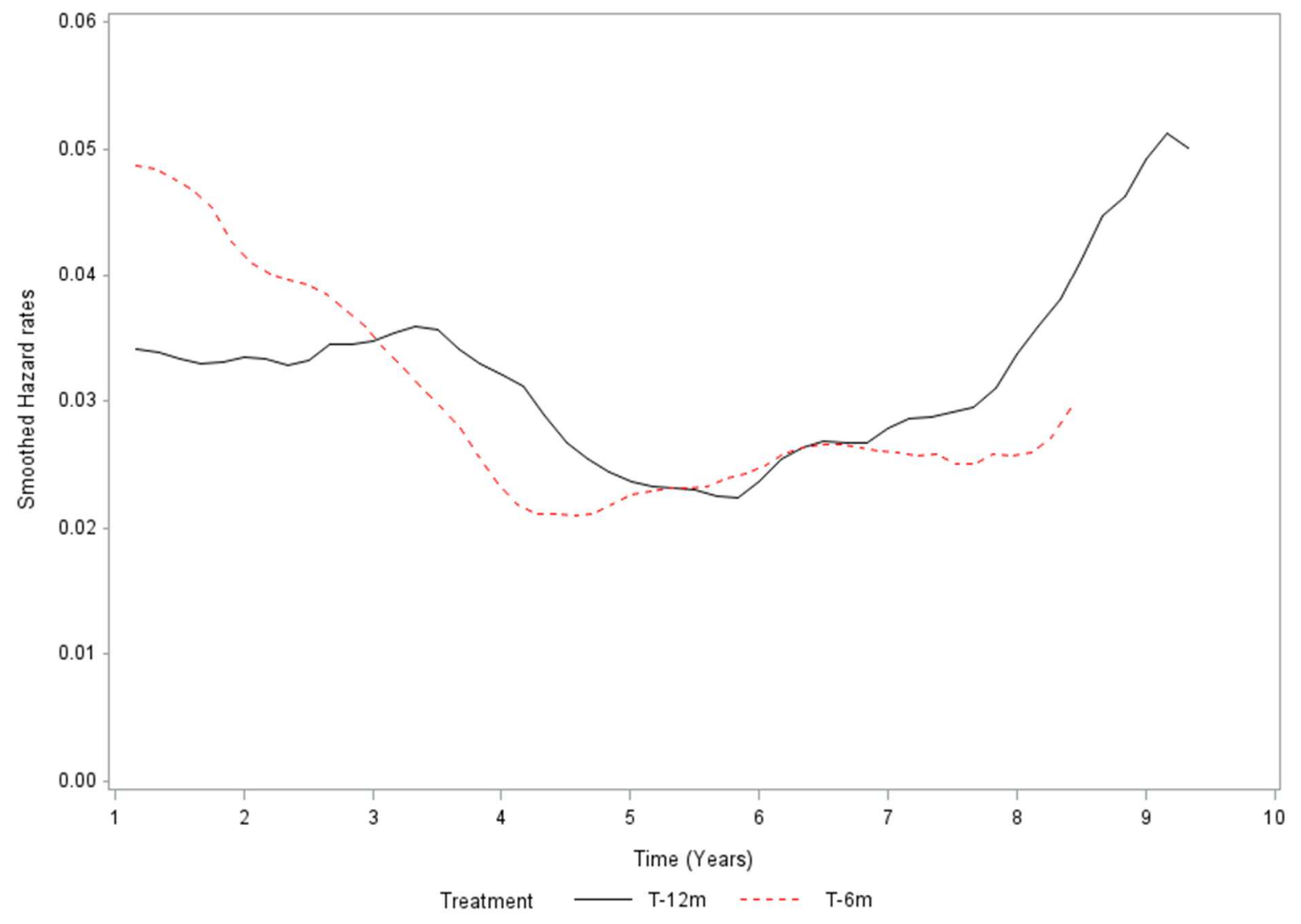


Figure 2E: Smoothed hazard ratios over time for overall survival events

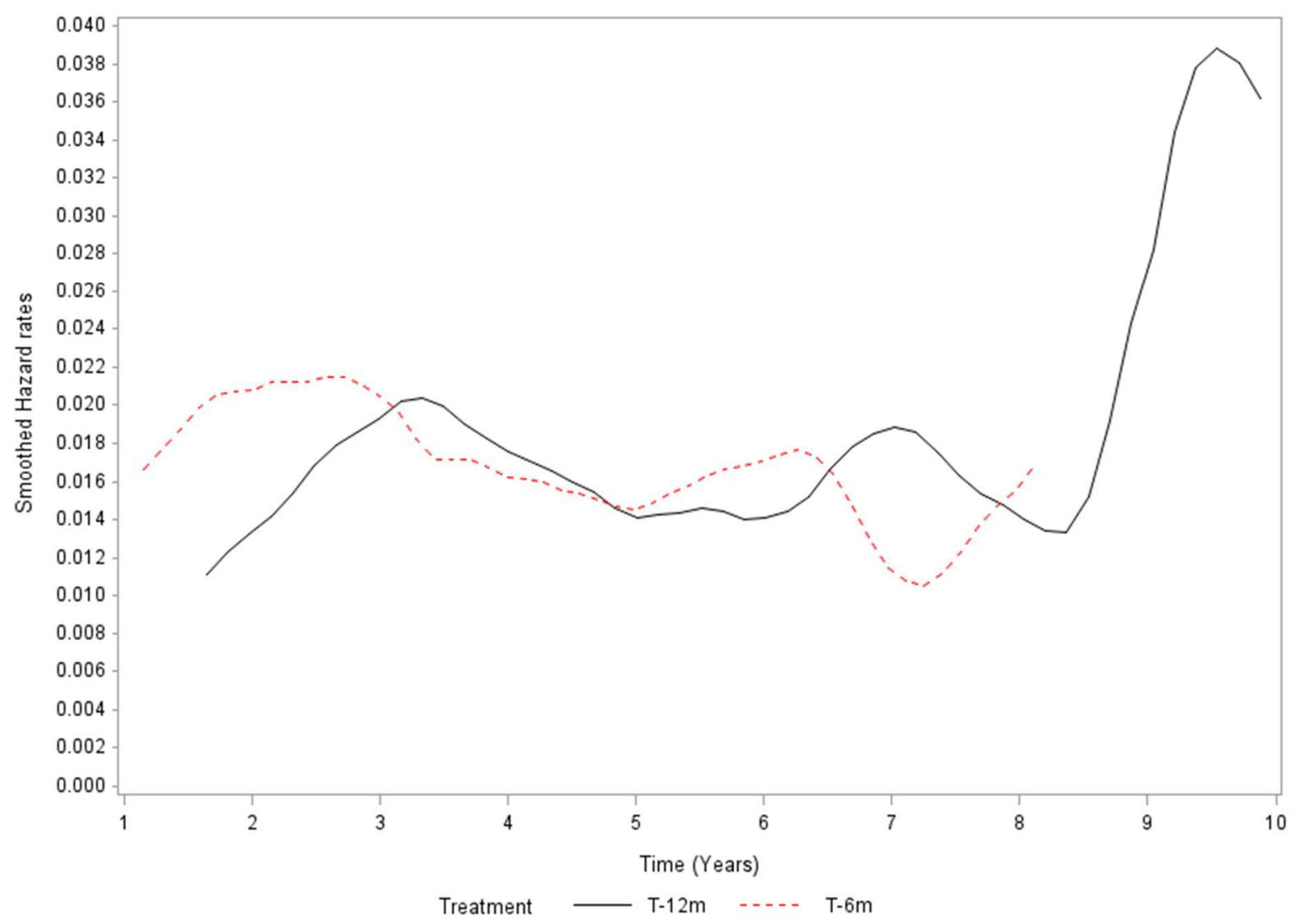


Figure 2F: Smoothed hazard ratios over time for metastasis-free survival events

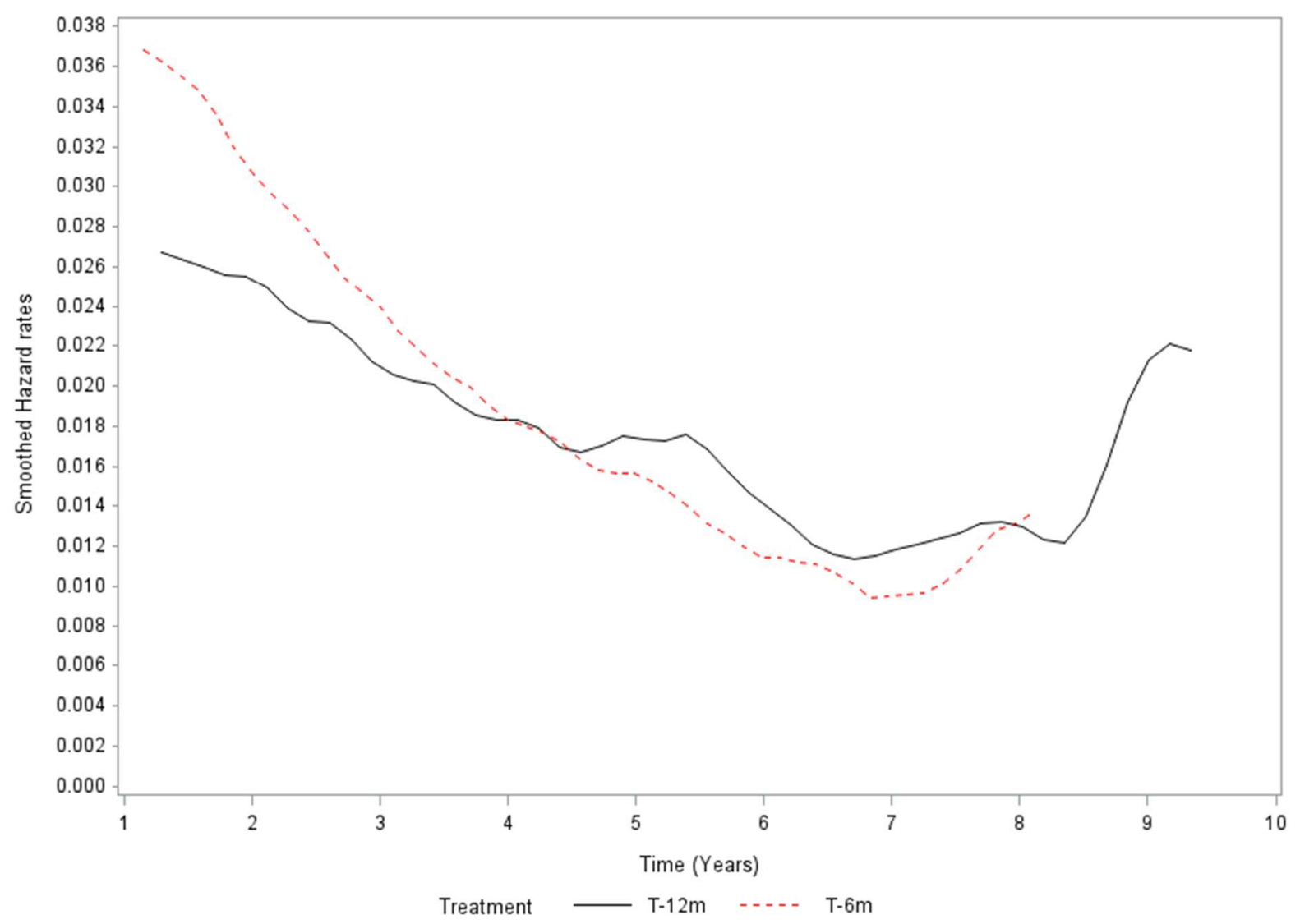


Figure 3: Univariate forest plot for disease-free survival

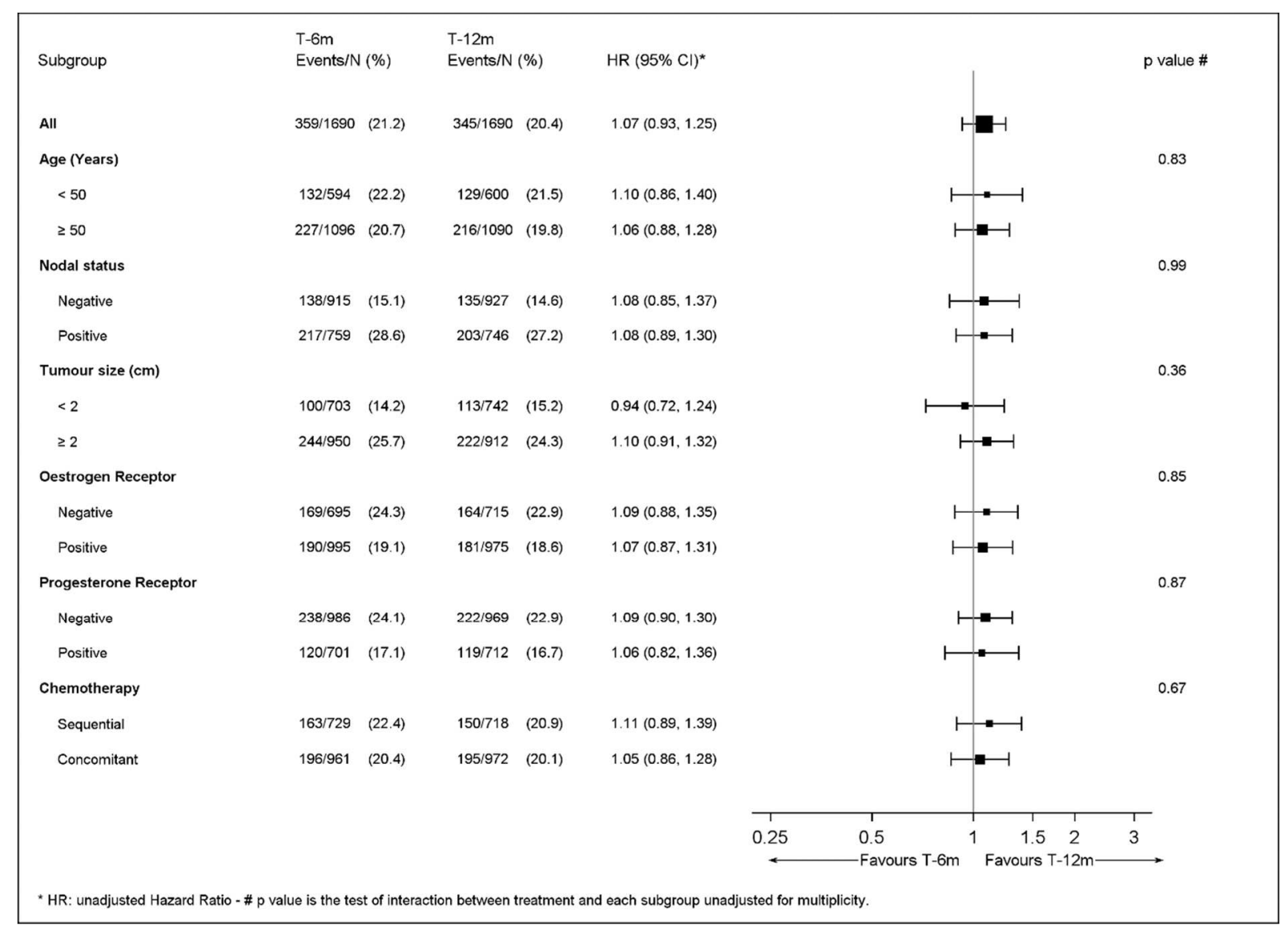



